NP-009

\section{THE IMPACT OF TNF $\alpha$ INHIBITORS ON GLUCOCORTICOIDS USE AMONG PATIENTS WITH ARTHRIDIS}

${ }^{1} \mathrm{R}$ Hafthorsdottir, ${ }^{2} \mathrm{AL}$ Gunnarsdottir, ${ }^{3} \mathrm{TJ}$ Love, ${ }^{4} \mathrm{G}$ Gerdur, ${ }^{5} \mathrm{~B}$ Gudbjornsson, on behalf of ICEBIO. 'Faculty of Pharmaceutical Sciences, The University of Iceland, Reykjavik, Iceland; ${ }^{2}$ Faculty of Pharmaceutical Sciences, The University of Iceland and Hospital Pharmacy, The University Hospital of Iceland, Reykjavik, Iceland; ${ }^{3}$ Faculty of Medicine, The University of Iceland and Department of Education, Research and Development, The University Hospital of Iceland, Reykjavik, Iceland; ${ }^{4}$ Department of Rheumatology, The University Hospital of Iceland, Reykjavik, Iceland; ${ }^{5}$ Faculty of Medicine, The University of Iceland and Centre for Rheumatology Research, The University Hospital of Iceland, Reykjavik, Iceland

\subsection{6/ejhpharm-2020-eahpconf.467}

Background and importance Glucocorticoids (GC) use among patients with arthritis is common, but due undesirable side effect it is vital to minimize their use as possible. The introduction of TNF $\alpha$ inhibitors has been a breakthrough in the treatment of arthridis leading to remission for many patients. In the literature there is however scarce information regarding the impact of TNF $\alpha$ inhibitors on GC use among these patients.

Aim and objectives To explore oral GC use in patients with rheumatoid arthritis (RA), psoriatic arthritis (PsA) and ankylosing spondylitis (AS) before and after initiation of treatment with TNF $\alpha$ inhibitors (TNFi).

The aim was also to evaluate if those patients on long term GC treatment were receiving adequate preventive osteoporosis treatment and how treatment with TNFi affect the use of topical steroids in patients with PsA.

Materials and methods Clinical data on patients with RA, PsA and AS which initiated TNFi therapy with etanercept, infliximab, adalimumab or golimumab for the first time between 2005-2015 was collected from the ICEBIO registry, a nationwide register on all patients treated with biologic drugs due to rheumatologic disorders in Iceland. Five controls were randomly selected from the Icelandic Medicine Database in Iceland (IMD) and matched to each patient in relation to age, sex and time frame. The use of oral GC, topical steroids and bisphosphonate was collected from IMD for period of four years, two years period before and two years period after the initiation of TNFi. The use was then evaluated in number of individuals, number of prescriptions and DDD (Defined Daily Dose).

Results 621 patients with RA, PsA or AS received 2630 prescriptions (3105 controls received 1337 prescriptions) for GC during the research period. The GC use varies between patient groups. The total GC use (DDD) doubled over the two-year period prior to the TNFi treatment, but decreased sharply after the initiation of TNFi. The number of individuals on GC decreased by one third after initiating TNFi therapy and the majority of those who continued GC treatment were patients with RA. $38 \%$ of those on long term GC treatment $(<7.5 \mathrm{mg} /$ day for three months) were receiving adequate preventive treatment for osteoporosis. The use of topical steroids decreased by half among PsA patients and one third discontinued the treatment after initiating TNFi.

Conclusion and relevance TNFi therapy does impact GC use among patients with arthritides, however large portion of RA patients are still on GS two years after initiate TNFi therapy. Better osteoporosis treatment is warranted for these patients.

\section{NP-010 CLINICAL AND PHARMACOKINETIC RESULTS AFTER THE SWITCH TO INFLIXIMAB BIOSIMILAR IN INFLAMMATORY BOWEL DISEASE: 2 YEARS OF REAL-LIFE EXPERIENCE}

${ }^{1} \mathrm{~N}$ Martín-Gutiérrez, ${ }^{1} \mathrm{JG}$ Sánchez-Hernández, ${ }^{1} \mathrm{~N}$ Rebollo-Díaz, ${ }^{2} \mathrm{~A}$ Fernández-Pordomingo, ${ }^{2} J F$ Muñoz-Núñez, ${ }^{1} V$ Recarey-Gerpe, ${ }^{1} M J$ Otero-López. 'Pharmacy Service, Complejo Asistencial Universitario de Salamanca; ${ }^{2}$ Gastroenterology Service, Complejo Asistencial Universitario de Salamanca

\subsection{6/ejhpharm-2020-eahpconf.468}

Background Debate on the use of biosimilars focuses on the therapeutic efficacy and safety of switching between biosimilars and their reference products.

Purpose To determine the clinical results and pharmacokinetic behaviour of switching from originator infliximab to biosimilar in patients with inflammatory bowel disease (IBD) over 2 years.

Materials and methods Prospective, longitudinal study (April 2017-March 2019). Patients with ulcerative colitis (UC) or Crohn's disease (CD) treated with originator infliximab (Remicade) and changed to biosimilar (CT-P13) were included.

The following outcome variables were defined: Clinical Remission $(\mathrm{CR})=$ Harvey Bradshaw index $<5$ in $\mathrm{CD}$ or partial Mayo index $<3$ in $\mathrm{CU}$; Endoscopic Remission (ER)= mucosa healing with absence of ultrasound activity; Biochemical Remission $(B R)=$ fecal calprotectin $<100 \mathrm{mg} / \mathrm{Kg}$.

Infliximab serum concentrations were determined by ELISA and pharmacokinetic parameters (volume of distribution (Vd) and clearance (CL)) were estimated by Bayesian population pharmacokinetics analysis.

Evaluation of variables was performed in four temporal sections: prior to the switch, immediately after, and again 8 months and 2 years after.

ANOVA test was used to compare pharmacokinetic parameters means and the percentage of patients who reached the outcome variables in the different temporal sections was calculated.

Results 42 patients $(55 \%$ women) were included, with a median [range] age of 42 [18-70] years, 10 diagnosed of UC and 32 of CD.

Prior to the switch, 93\% of the patients presented CR and $\mathrm{ER}$, and 95\% BR. These results were identical immediately after switching. Eight months after the switch, 93\% of the patients presented $\mathrm{CR}$ and $88 \% \mathrm{ER}$ and BR. At the end of the two years' follow-up, 97\% presented CR and 92\% ER and BR.

Regarding pharmacokinetic behaviour, there were no significant differences between the average values of CL estimated in the different sections, which were: $0.393 \mathrm{~L} / \mathrm{d} ; 0.392 \mathrm{~L} / \mathrm{d}$; $0,395 \mathrm{~L} / \mathrm{d}$ and $0,390 \mathrm{~L} / \mathrm{d}(\mathrm{p}=0.91)$, nor among the $\mathrm{Vd}$, whose results were: $5.25 \mathrm{~L} ; 5.25 \mathrm{~L} ; 5.24 \mathrm{~L}$ and $5.28 \mathrm{~L}(\mathrm{p}=0.93)$, respectively.

Conclusion After switching from infliximab originator to biosimilar in a real cohort of IBD patients, no changes in clinical outcomes or pharmacokinetic behaviour were observed over 2 years, which supports the switch in clinical practice. 